\title{
Simulación condicional de variables regionalizadas y su aplicación al comportamiento de la porosidad efectiva en un yacimiento fracturado-poroso
}

\author{
José Quintín Cuador-Gil $^{1 *}$ y Arelys Quintero-Silverio ${ }^{2}$ \\ 1 Departamento de Informática, ${ }^{2}$ Departamento de Matemática, \\ Universidad de Pinar del Río, Martí, No. 270, Pinar del Río, 20100, Cuba. \\ * cuador@netupr.upr.edu.cu
}

\begin{abstract}
Resumen
En el campo de las geociencias es muy frecuente encontrar variables que se manifiestan distribuidas espacialmente. Para el estudio de estas variables son usados actualmente diversos procedimientos de estimación y/o simulación. Esto es, a partir de un conjunto de muestras tomadas en localizaciones del dominio en que se manifiesta un fenómeno a estudiar y consideradas representativas de su realidad, que por lo general es siempre desconocida, estos procedimientos permiten su descripción o caracterización con dos fines diferentes: el primero es proporcionar valores estimados en localizaciones de interés y el segundo, generar valores que en conjunto presenten iguales características de dispersión que los datos originales.

Particularmente en la geología y la minería ha tenido un amplio uso la estimación propuesta por la geoestadística a través del procedimiento kriging. Esta estimación es sin lugar a dudas la mejor posible, pues aporta el mejor estimador lineal insesgado, y una representación de ello es el número creciente de aplicaciones en Ciencias de la Tierra donde es utilizada actualmente: industrias minera y del petróleo, estudios ambientales, cartografía, climatología, etc. Este método, de acuerdo a las condiciones con que es obtenido, así como la amplia gama de interpoladores conocidos, muestran una imagen suave de la realidad que se estudia, sin tener en cuenta las fluctuaciones siempre presentes en la práctica. Una alternativa para esta situación es simular el comportamiento de las variables analizadas. Considerando las variables como funciones aleatorias, se puede obtener una de las posibles realizaciones del fenómeno con las mismas características de variabilidad y correlación espacial de los datos originales y por tanto de la realidad, características que son reveladas de la información disponible a través del cálculo de los semivariogramas experimentales y el ajuste de modelos teóricos. La simulación no debe ser considerada superior a la estimación, sino como una alternativa en el grado de descripción que se desee realizar. Los objetivos de estos procedimientos son diferentes, pero en muchos casos el análisis de sus resultados paralelamente pueden contribuir a enriquecer la caracterización que se desarrolle en el estudio de variables regionalizadas.

En este artículo se presentan los elementos fundamentales de la teoría de la simulación condicional, el cual usa en el proceso de condicionamiento al kriging. Se introduce un algoritmo de simulación en una dimensión que puede ser extendido a dos y tres dimensiones. Finalmente se muestra un ejemplo de aplicación en la simulación de la porosidad efectiva en el horizonte productivo Tobas Gruesas del yacimiento fracturado poroso Pina, de la provincia Ciego de Ávila, Cuba.

Palabras clave: Geoestadística, semivariograma, Kriging, simulación condicional, función aleatoria, algoritmo.
\end{abstract}

\begin{abstract}
In geosciences, it is very frequent to find spatially distributed variables. Nowadays, different estimation and/or simulation procedures are used to study this type of variables. From a set of samples taken from some location over the domain of the phenomenon, which are considered representative of the reality (reality that generally is always unknown), these procedures allow its description or characterization in two different ways: the first one gives estimated values in the location of interest where no samples were taken, and the second one generates values with the same
\end{abstract}


characteristic dispersion of the original data.

Particularly in geology and mining, the kriging estimation proposed by geostatistics has been widely used. This is certainly the best possible estimation, as it provides the Best Linear Unbiased Estimator. This fact is confirmed by the increasing number of geostatistical applications that are presently carried out in earth sciences, especially in the mining industry, as well as in oil industry, environmental studies, cartography, climatology, etc. The geostatistical estimation, according to the mathematical condition from which it is obtained, gives a smooth image of the elements under study, in the same way as the wide group of other known interpolators. This estimation shows no fluctuation of its contents. An alternative for this situation is to simulate the behavior of the variable. Considering these variables as random functions it is possible to obtain a realization with the same variability and correlation characteristics of the original data, which can be used to represent the subject under investigation. These characteristics are revealed from the available information by the calculation of semivariograms and the fitting of theoretical models. The simulation process should not be considered better than the estimation process, but as an alternative to obtain the level of description needed. The objectives of these procedures are different, but they together could improve the knowledge of the regionalized variable.

In this paper, we present the fundamental elements of the conditional simulation theory that uses kriging in the conditioning process, and introduce an algorithm of simulation in one dimension, which can be extended for two and three dimension. Finally, we show an example of the simulation of the effective porosity in the productive horizon Tobas Gruesas in the fractured-porous reservoir Pina situated in the province of Ciego de Ávila in Cuba.

Keywords: Geostatistics, semivariogram, Kriging, conditional simulation, random function, algorithm.

\section{Introducción}

Son muchos los ejemplos en las Ciencias de la Tierra que conducen al tratamiento de variables distribuidas espacialmente, por ejemplo la estimación de recursos minerales, la caracterización de parámetros geofísicos en yacimientos de petróleo, la evaluación de variables ambientales, etc. Para el estudio de estos datos, que tienen asociada siempre una posición en el espacio y conocidos como variables regionalizadas (Matheron, 1970), se usan actualmente métodos geomatemáticos, dentro de los cuales la geoestadística ha jugado en las tres últimas décadas un papel importante y ha probado su superioridad entre tantos métodos de estimación de reservas en numerosos tipos de minas (Armstrong y Carignan, 1997). Su extensión a otros campos de aplicación, aunque reciente, ha mostrado valiosos resultados, como se puede apreciar en los trabajos de Galli (1997) en la industria del petróleo y Myers (1988) en estudios ambientales, además de una amplia gama de trabajos de otros autores en diferentes campos de aplicación de las geociencias.

La geoestadística se define de forma general como el estudio de las variables numéricas distribuidas en el espacio (Chauvet, 1994). Este método exige para su aplicación el cumplimiento de ciertas hipótesis de estacionaridad (Journel y Huijbregts, 1978; Armstrong y Carignan, 1997), las cuales pueden ser verificadas a través del análisis exploratorio de los datos experimentales. Su estimador, el kriging, término creado por G. Matheron en 1962, tiene como objetivo encontrar el Mejor Estimador Lineal Insesgado a partir de la información disponible (ChicaOlmo, 1987). En efecto, el estimador obtenido ( $\left.Z^{*}\right)$ consiste en una combinación lineal del producto de una ponderación de pesos asociados a cada localización muestreada por el correspondiente valor $\mathrm{Z}$ del fenómeno estu- diado, observando dos condiciones fundamentales: 1) que el estimador sea insesgado: $E\left[Z^{*}-Z\right]=0$; $y$ 2) que la varianza $\operatorname{Var}\left[Z^{*}-Z\right]$ sea mínima.

La estimación obtenida de esta forma, al igual que otros interpoladores, proporciona una imagen suave o lisa de la realidad, pero como en muchas ocasiones no es suficiente conocer sólo los valores estimados, sería conveniente una representación capaz de reflejar las fluctuaciones siempre presentes en la práctica, lo que es posible utilizando la dispersión del fenómeno estudiado (Journel, 1974). Como las variables regionalizadas son consideradas en geoestadística como una función aleatoria, es posible obtener una realización particular de ésta (Journel y Huijbregts, 1978), es decir, una simulación que no sea una representación suave o lisa de la realidad, sino más bien una de las posibles realizaciones del fenómeno estudiado, de modo que presente la misma dispersión, o lo que es igual, las mismas características de variabilidad y correlación espacial encontrada en los datos experimentales, y por tanto que en la realidad desconocida.

En este artículo se presentan los elementos fundamentales de la teoría de la simulación geoestadística, la cual desde su introducción por Matheron (1962) y su desarrollo por Guibal (1972) y Journel (1974) ha jugado un importante papel en las aplicaciones de la geoestadística (Lantuéjoul, 1997); en la simulación condicional de yacimientos mineros (Journel, 1974; Chica-Olmo, 1987), en la simulación de litofacies en la industria del petróleo (Le Loc'h y Galli, 1997); en la representación de modelos de yacimientos petroleros (Allard, 1993); en la modelación de procesos hidrogeológicos (Dietrich, 1995); en la presentación de procesos geofísicos (Christakos y Panagopoulos, 1992), etc. Se presenta además un algoritmo de simulación para una dimensión que puede ser extendido a dos y tres dimensiones, así como un ejemplo sobre 
la caracterización de la porosidad efectiva en el horizonte Tobas Gruesas del yacimiento petrolífero Pina, de tipo fracturado-poroso, de la provincia Ciego de Ávila, Cuba.

\subsection{Estimación o Simulación, dos alternativas}

La estimación tiene como objetivo proporcionar en cada localización a estimar un valor $Z^{*}(x)$, lo más próximo posible del valor real y desconocido $\mathrm{Z}(\mathrm{x})$ del fenómeno estudiado. La estimación no reproduce la dispersión de los datos reales, más bien subestima en general esta dispersión (Journel, 1974). Por el contrario, la simulación, o mejor la simulación condicional de la geoestadística, reproduce los dos primeros momentos experimentales (media y covarianza $\mathrm{C}(\mathrm{h})$ o Semivariograma $\gamma(\mathrm{h}))$ de los datos reales, así como el histograma, es decir, se reproducen las principales características de dispersión del fenómeno real de acuerdo a la información disponible. Ahora, en cada localización, el valor simulado $\mathrm{Z}_{\mathrm{S}}(\mathrm{x})$ no es el mejor estimador de $\mathrm{Z}(\mathrm{x})$ que es posible obtener.

Si nos hacemos la pregunta: ¿en qué son diferentes la simulación y la estimación?, podemos responder que sus diferencias están relacionadas con sus objetivos, los cuales en general no son compatibles (Journel, 1974), porque mientras en la estimación se obtienen valores insesgados con respecto a la realidad que es sólo conocida en algunos puntos experimentales, la simulación proporciona valores que reproducen las fluctuaciones que se presentan en la realidad a partir de la información que brindan estos puntos conocidos, por lo que dependiendo de los intereses en una aplicación o investigación se utilizará una u otra. A partir de lo anterior podemos decir que estos procedimientos son dos alternativas que se pueden utilizar conjuntamente para caracterizar las variables regionalizadas.

\section{Teoría de la Simulación Condicional}

Consideremos los datos experimentales $\mathrm{Z}\left(\mathrm{x}_{\mathrm{i}}\right)$; con $\mathrm{i}=1, \ldots, \mathrm{n}$; con media $\mathrm{m}$ y covarianza $\mathrm{C}(\mathrm{h})$ o Semivariograma $\gamma(\mathrm{h})$. En una estimación por kriging se obtiene un valor $\mathrm{Z}^{*}(\mathrm{x})$ por cada localización a estimar, el cual, según las condiciones con que fue construido este procedimiento, es el mejor valor posible, es decir, es un valor insesgado que se produce de acuerdo a las características de continuidad espacial de la información disponible. Ahora, como no existe ningún método, por muy sofisticado que sea, que proporcione valores exactos de una realidad desconocida, sólo es posible plantear que el valor estimado por kriging $\mathrm{Z}_{\mathrm{K}} *(\mathrm{x})$ es aproximado al valor real y desconocido $\mathrm{Z}(\mathrm{x})$.

No es difícil comprender que estos valores son diferentes en la cantidad $\left[\mathrm{Z}(\mathrm{x})-\mathrm{Z}_{\mathrm{K}} *(\mathrm{x})\right]$, de modo que la aproximación anterior se puede completar de forma exacta como:

$$
\mathrm{Z}(\mathrm{x})=\mathrm{Z}_{\mathrm{K}} *(\mathrm{x})+\left[\mathrm{Z}(\mathrm{x})-\mathrm{Z}_{\mathrm{K}} *(\mathrm{x})\right]
$$

Pero la diferencia $\left[\mathrm{Z}(\mathrm{x})-\mathrm{Z}_{\mathrm{K}} *(\mathrm{x})\right]$ no se conoce, porque no se poseen los valores de las localizaciones no muestreadas, sólo es posible obtener su valor en las localizaciones donde fueron medidos valores experimentales, los cuales son nulos debido a que el kriging es un interpolador exacto (Matheron, 1970), es decir, la estimación en las localizaciones muestreadas coincide con el valor real medido.

El problema consiste entonces en encontrar un modo de obtener la diferencia $\left[\mathrm{Z}(\mathrm{x})-\mathrm{Z}_{\mathrm{K}} *(\mathrm{x})\right]$, lo que sólo es posible mediante una simulación. La geoestadística propone este procedimiento generando primero valores de una función aleatoria $\mathrm{Z}_{\mathrm{S}}(\mathrm{x})$ a partir de las características de variabilidad y correlación espacial de la información experimental disponible $\mathrm{Z}\left(\mathrm{x}_{\mathrm{i}}\right)$, a la cual posteriormente son condicionados (Lantuéjoul, 1998; Rivoirard 1998).

Para ilustrar lo planteado hasta aquí, consideremos que los valores experimentales $\mathrm{Z}\left(\mathrm{x}_{\mathrm{i}}\right)$ se distribuyen espacialmente según la Figura 1, los cuales pueden distribuirse regular o irregularmente, además pueden existir combinaciones de estas formas. Se dispone además de una red con un espaciamiento tal que abarque todas las localizaciones que se desean simular (Figura 2), colocada sobre la misma zona de estudio.

Existiendo un conjunto de localizaciones compuestas por los puntos de la red y aquellos donde se conoce un valor experimental, en cada localización de este conjunto se generan valores de una función aleatoria $\mathrm{Z}_{\mathrm{S}}(\mathrm{x})$ a partir de las características de variabilidad obtenida de la información inicialmente disponible, este proceso se conoce como simulación no condicional, para lo cual

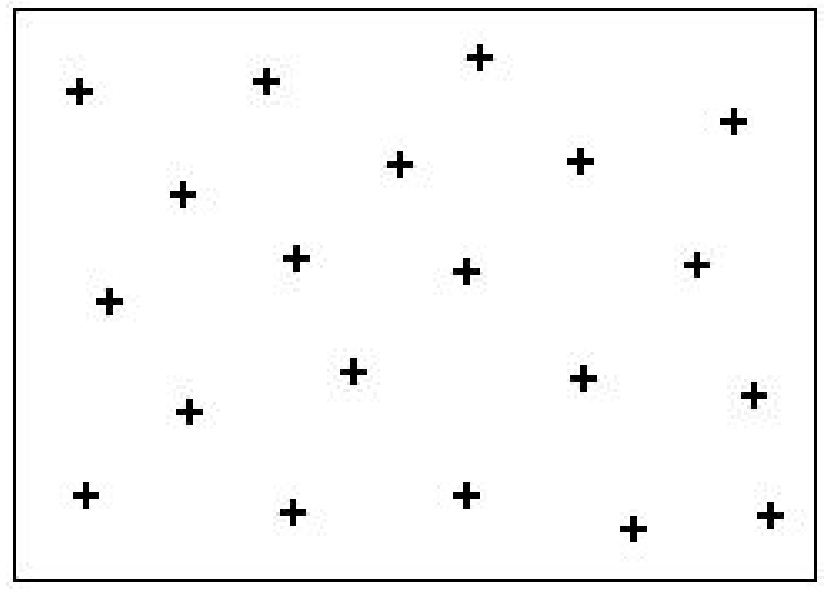

Figura 1. Localizaciones muestreadas. 


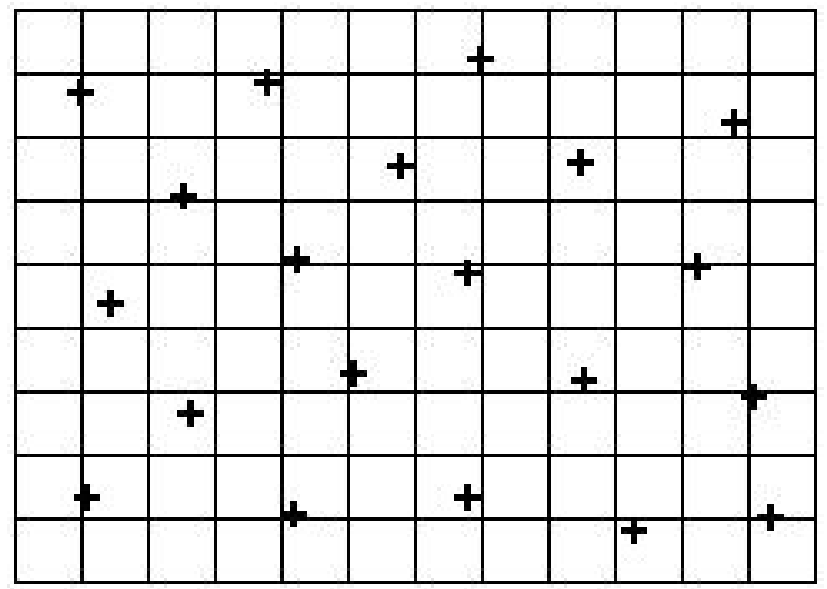

Figura 2. Red de puntos a simular.

existen diferentes métodos (Journel, 1974; Journel y Huijbregts, 1978; Lantuéjoul, 1994; Lantuéjoul, 1998; Rivoirard, 1998; Deutsch y Journel, 1998).

Posteriormente, con el objetivo de condicionar la simulación a los valores experimentales, se realiza la estimación por kriging de cada punto de la red a partir del conjunto de valores simulados de forma no condicional en las localizaciones donde se conocen datos experimentales, obteniéndose los valores $Z^{*}{ }_{\mathrm{SK}}(\mathrm{x})$. Ahora, a partir de estos valores y los simulados de forma no condicional $Z_{S}(x)$, se puede simular la diferencia $[Z(x)$ $\left.\mathrm{Z}^{*}{ }_{\mathrm{K}}(\mathrm{x})\right]$ como $\left[\mathrm{Z}_{\mathrm{S}}(\mathrm{x})-\mathrm{Z}_{\mathrm{SK}}(\mathrm{x})\right]$, por lo que la expresión (1) se puede sustituir por:

$$
\mathrm{Z}_{\mathrm{SC}}(\mathrm{x})=\mathrm{Z}_{\mathrm{K}} *(\mathrm{x})+\left[\mathrm{Z}_{\mathrm{S}}(\mathrm{x})-\mathrm{Z}_{\mathrm{SK}} *(\mathrm{x})\right]
$$

donde:

$\mathrm{Z}_{\mathrm{SC}}(\mathrm{x})$ es el valor simulado en el punto $\mathrm{x}$ por la simulación condicional.

$Z_{\mathrm{K}} *(\mathrm{x})$ es el valor estimado en el punto $\mathrm{x}$ por el procedimiento kriging, a partir de los datos

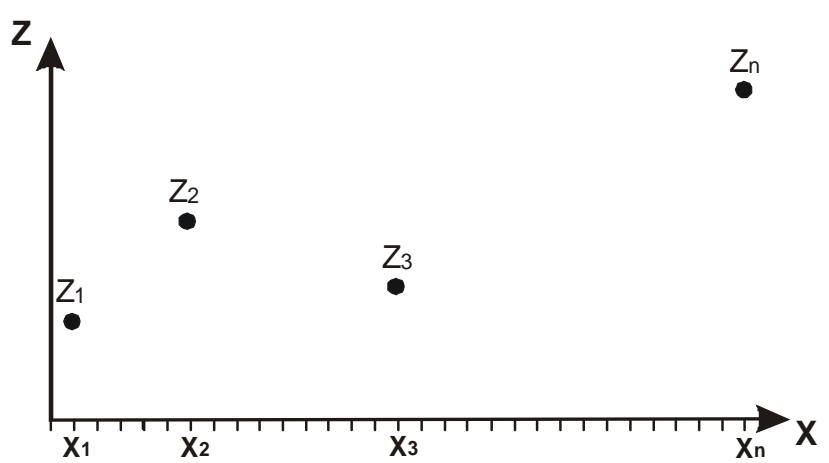

Figura 3. Distribución de datos medidos experimentalmente y localizaciones propuestas a simular. experimentales.

$\mathrm{Z}_{\mathrm{S}}(\mathrm{x})$ es el valor simulado en el punto $\mathrm{x}$ por la simulación no condicional.

$\mathrm{Z}_{\mathrm{SK}}{ }^{*}(\mathrm{x})$ es el valor estimado en el punto $\mathrm{x}$ por el procedimiento kriging, a partir de los valores simulados no condicionalmente en las localizaciones correspon-dientes a los datos experimentales.

Se obtienen de esta forma valores simulados, uno por cada localización de la red, que son en conjunto una realización particular de la función aleatoria de las tantas posibles que pueden ser generadas, de la cual la realidad desconocida lo es también, pero que reflejan las mismas características de variabilidad y correlación espacial que esta realidad.

\section{Algoritmo de Simulación Condicional}

Consideremos el caso en una dimensión para facilitar la explicación. Se poseen $\mathrm{Z}\left(\mathrm{x}_{\mathrm{i}}\right)$; con $\mathrm{i}=1, \ldots, \mathrm{n}$ datos experimentales. En la Figura 3 se representa su distribución espacial en un gráfico de los valores medidos $\mathrm{Z}$ contra su posición x. Es importante señalar aquí que los datos iniciales pueden distribuirse de forma regular o irregular, en el ejemplo tomamos el segundo caso.

1. Mediante un análisis estructural es posible obtener un modelo que represente las características de variabilidad y correlación espacial del fenómeno estudiado.

2. Se proponen una serie de localizaciones en la misma zona de estudio, donde se desee simular el fenómeno analizado (Figura 3). En dos dimensiones se tendría una red de puntos, y en tres dimensiones una distribución regular de puntos o bloques en el interior de un volumen.

3. Se estima un valor en cada localización propuesta por kriging a partir de los datos experimentales, obteniéndose los valores $Z_{\mathrm{K}} *(\mathrm{x})$ (Figura 4). Como se

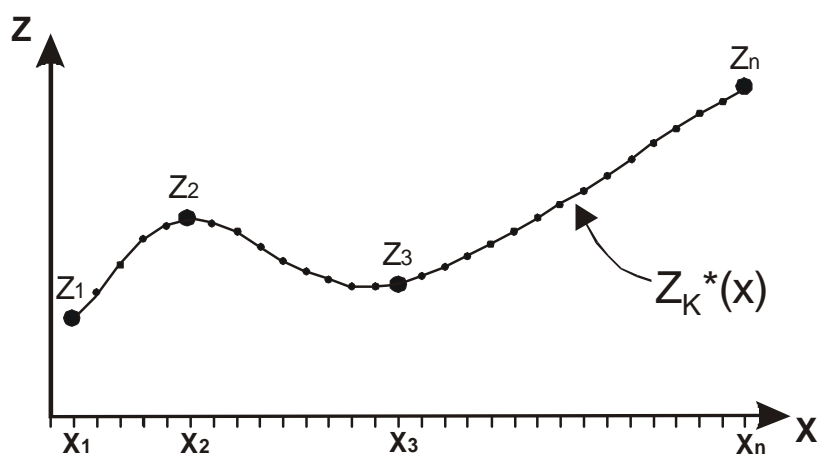

Figura 4. Valores $\mathrm{Z}_{\mathrm{K}}{ }^{*}(\mathrm{x})$, obtenidos por kriging a partir de los datos experimentales. 


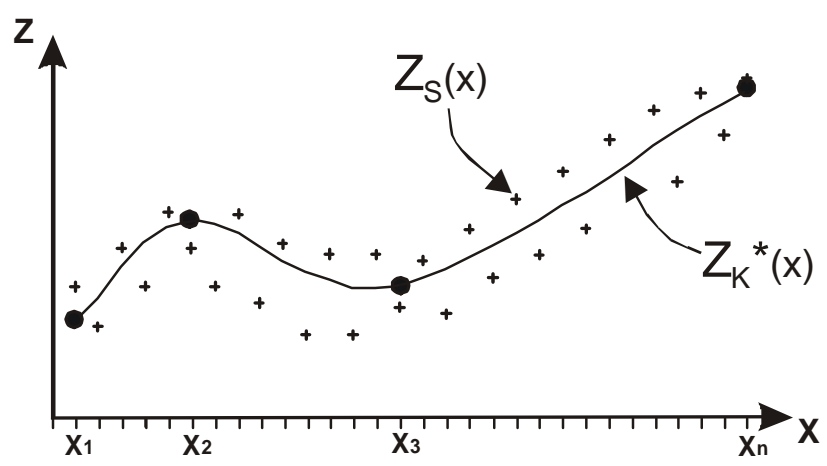

Figura 5. Valores $Z_{S}(x)$, simulados no condicionalmente.

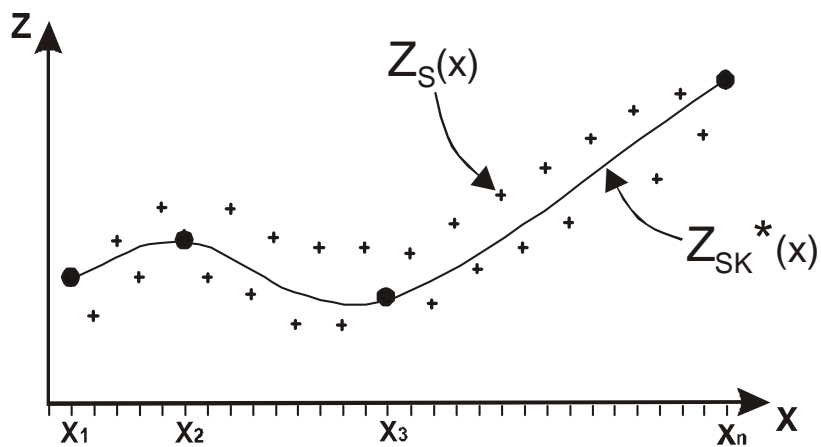

Figura 6. Valores $\mathrm{Z}_{\mathrm{SK}}{ }^{*}(\mathrm{x})$, obtenidos por kriging a partir de los datos simulados en las localizaciones muestreadas.

puede apreciar, la estimación realizada es una representación suave de una realidad desconocida.

4. Se generan simulaciones no condicionadas en las localizaciones propuestas y en las localizaciones donde se poseen datos experimentales, a partir de las características de variabilidad obtenidas en el primer paso, obteniéndose los valores $\mathrm{Z}_{\mathrm{S}}(\mathrm{x})$. (Figura 5). Note que si los puntos experimentales son regulares, las localizaciones muestreadas coinciden con sus correspondientes localizaciones propuestas en la simulación.

5. Se estiman nuevamente por kriging las localizaciones propuestas a simular, pero esta vez a partir de los valores simulados en las localizaciones correspondientes a los datos experimentales, obteniéndose los valores $\mathrm{Z}_{\mathrm{SK}}{ }^{*}(\mathrm{x})$ (Figura 6).

6. Se calcula la diferencia $\left[\mathrm{Z}_{\mathrm{S}}(\mathrm{x})-\mathrm{Z}_{\mathrm{SK}} *(\mathrm{x})\right]$, para cada localización de la red propuesta (Figura 7).

7. Se suma a la estimación obtenida en el paso 3 (Figura 4), la correspondiente diferencia calculada en el paso anterior (Figura 7), condicionándose de esta forma la simulación y obteniéndose el valor simulado $\mathrm{Z}_{\mathrm{SC}} *(\mathrm{x})$ (Figura 8).

Los pasos anteriores pueden ser generalizados sin dificultad a dos o tres dimensiones.

\section{Simulación de parámetros geofísicos, un ejemplo}

Los estudios geológicos y geofísicos efectuados en Cuba en estos últimos años demuestran perspectivas favorables para la localización de nuevos yacimientos de petróleo, existiendo numerosos datos geológicos y geofísicos, tanto de superficie como en pozos profundos, que permiten realizar análisis probabilísticos y geoestadísticos, tanto en aquellas zonas de yacimientos en producción como en zonas de probable existencia, lo cual es una premisa imprescindible para la elaboración de los modelos matemáticos. Se presenta como ejemplo la caracterización de la porosidad efectiva en algunos de los horizontes del yacimiento Pina, municipio de Majagua, provincia de Ciego de Ávila.

El campo Pina fue descubierto en 1990 y cubre un área de alrededor de $3.03 \mathrm{~km}^{2}$, con una forma alargada de noroeste a sur, esto es $2.17 \mathrm{~km}$ de largo y $1.48 \mathrm{~km}$ de ancho. El yacimiento tiene aproximadamente $600 \mathrm{~m}$ de espesor, creciendo desde los flancos, con $155 \mathrm{~m}$ en la parte más hundida de la estructura, hacia la cúpula con $400 \mathrm{~m}$. Sus características tectónicas, fallas y plegamientos, lo dotan de cierta complejidad. Su relieve está representado por una llanura con una altura media sobre el nivel del mar de 50m. El campo está constituido por rocas intrusivas y volcanogénicas. La porosidad tiene un

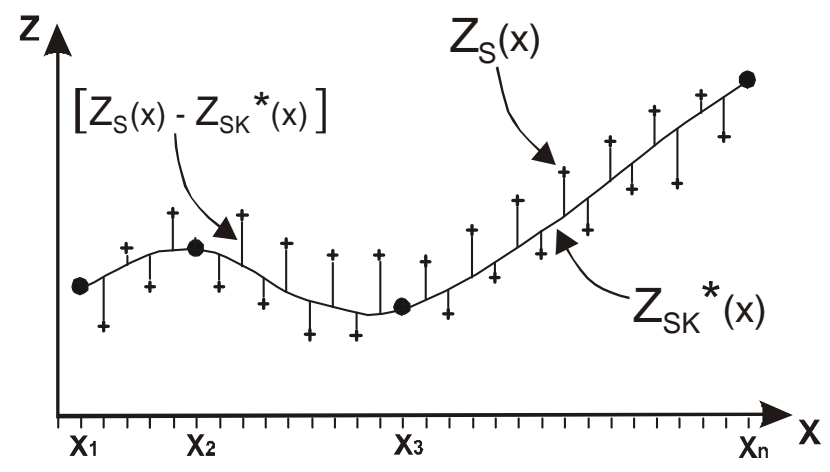

Figura 7. Diferencia $\left[\mathrm{ZS}(\mathrm{x})-\mathrm{ZSK}^{*}(\mathrm{x})\right]$ simulada.

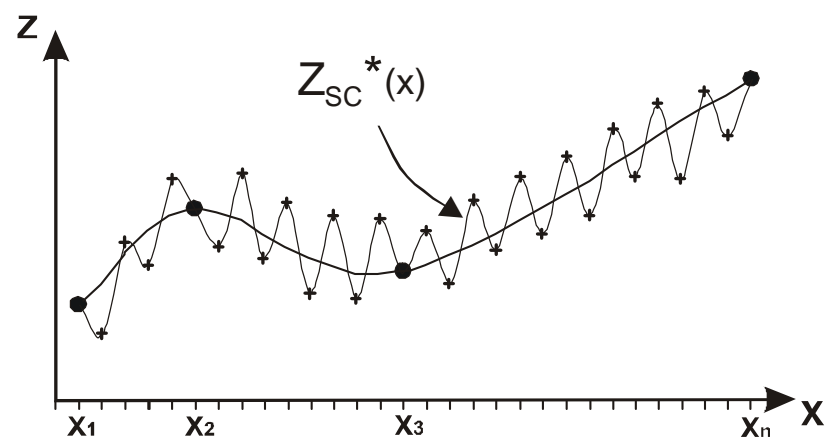

Figura 8. Valores $\mathrm{Z}_{\mathrm{SC}}{ }^{*}(\mathrm{x})$ de la simulación condicional. 
carácter secundario, preferentemente en fracturas que mantienen diversas direcciones, predominando la subvertical. Esta constitución geológica del campo refleja la existencia de un estilo tectónico similar al de las unidades tectonoestratigráficas presentes en la costa norte, o sea, pliegues sobrecorridos, divididos en bloques por fallas normales de desplazamiento y fallas inversas.

La capacidad gasopetrolífera de Pina se ubica en tres horizontes litológicos fundamentales denominados por Álvarez et al. (1996) como: Tobas Finas, Tobas Gruesas y Efusivos. Estos horizontes están relacionados con rocas derivadas del arco volcánico, siendo el de mayor peso productivo el correspondiente al corte volcánico sedimentario.

El horizonte Tobas Gruesas está constituido por tobas de grano grueso y brechas volcánicas, también de grano medio, mayores de $1 \mathrm{~mm}$ de diámetro. Estas rocas tienen dos texturas predominantes que son la lítica y la cristalino-lítica; en menor escala se presenta la textura cristalino-vítrea (principalmente para la granulometría más pequeña dentro del grupo). En este grupo predominan las rocas de composición basáltica, con intercalaciones menores de sedimentos. Se pueden encontrar fragmentos de calizas y areniscas con matriz tobácea. Con mayor frecuencia, se encuentran intercalados entre las tobas gruesas conglomerados tobáceos que se describen como rocas de grano muy grueso.

La información de partida está constituida por valores de porosidad efectiva obtenidos a través de la interpretación de registros geofísicos en 48 pozos, de los cuales 30 cortan el horizonte Tobas Gruesas (Figura 9).
Se comenzó realizando un análisis descriptivo de las variables, lo cual permite detectar la existencia valores anómalos y conocer la distribución estadística a la cual se ajusta la misma, a través de la producción de los histogramas y las pruebas de bondad de ajuste. La porosidad efectiva del horizontes Tobas Gruesas muestra una distribución bastante simétrica, con valores similares de la media y la mediana, el coeficiente de variación es menor que uno, la prueba Kolmogorov-Smirnov mostró que la distribución de los datos es normal para un nivel de significación del $5 \%$, por lo que la variable pertenece a una población homogénea unimodal.

Una vez descrita la información se procedió a realizar el análisis estructural, donde se calculó inicialmente el mapa de variograma, permitiendo obtener las posibles direcciones de anisotropía. Para el caso de la porosidad en Tobas Gruesas, se observó que muestra mayor continuidad espacial en la dirección noroeste, la cual coincide con el rumbo del yacimiento. Posteriormente fueron calculados los semivariogramas direccionales, utilizando un incremento de $200 \mathrm{~m}$, que es la distancia promedio entre las muestras contiguas y calculados hasta $1,500 \mathrm{~m}$, aproximadamente la mitad de las dimensiones del yacimiento a partir de donde el semivariograma deja de ser significativo y en las direcciones noroeste y noreste con tolerancias angulares de $45^{\circ}$. A continuación se ajustaron modelos teóricos, obteniéndose un modelo anidado compuesto por dos modelos esféricos con mesetas $2 \%^{2}$ y $2.25 \%^{2}$ y alcances de $660 \mathrm{~m}$ y $3,250 \mathrm{~m}$ de la dirección de mayor continuidad espacial y $532 \mathrm{~m}$ y $599 \mathrm{~m}$ en la dirección

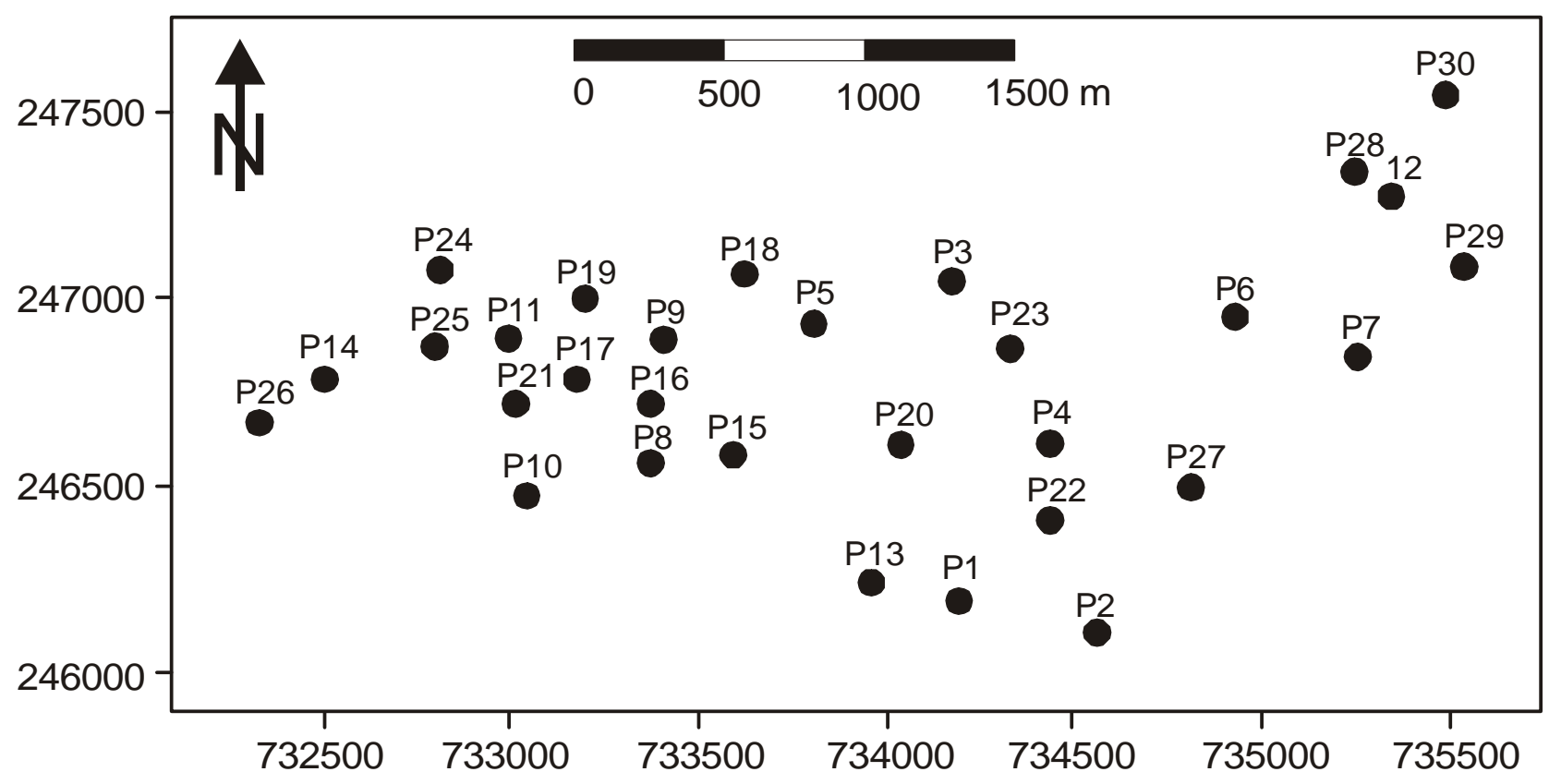

Figura 9. Mapa de localización de datos reales en el horizonte Tobas Gruesas del yacimiento Pina. 
perpendicular a la anterior, lo que se resume en la siguiente expresión:

$\gamma\left(h_{x}, h_{y}\right)=2$ Sph $(660,532)+2.25 \operatorname{Sph}(3,250,599)$, donde Sph representa el modelo esférico. Se utilizó el proceso de la validación cruzada para validar los modelos teóricos ajustados, reportando criterios aceptables de la media de los errores de estimación y la varianza de los errores estandarizados.

La estimación de la variable mostrada en este ejemplo se realizó utilizando el interpolador kriging, en una rejilla de $146 \times 74$ bloques con tamaño de $25 \times 25 \mathrm{~m}$, barriéndose todo el dominio y contorneándose posteriormente de acuerdo a la distribución de los datos experimentales. La estimación por kriging, como hemos dicho, proporciona una imagen suave de la realidad que se estudia. Se procede entonces a la simulación de la variable tratada a partir de las características de variabilidad y correlación espacial obtenidas en el análisis estructural y con las mismas dimensiones de la red de estimación. Para el procesamiento de la información fue utilizado el paquete GsLib: Geostatitical Library (Deutsch y Journel, 1998). En la Figura 10 se observan los mapas de estimaciones y simulaciones, donde se presenta el comportamiento de la variable porosidad efectiva" en el horizonte Tobas Gruesas, observándose una tendencia en la dirección noroestesureste que coincide con la dirección del rumbo del yacimiento y probablemente con la línea de máxima

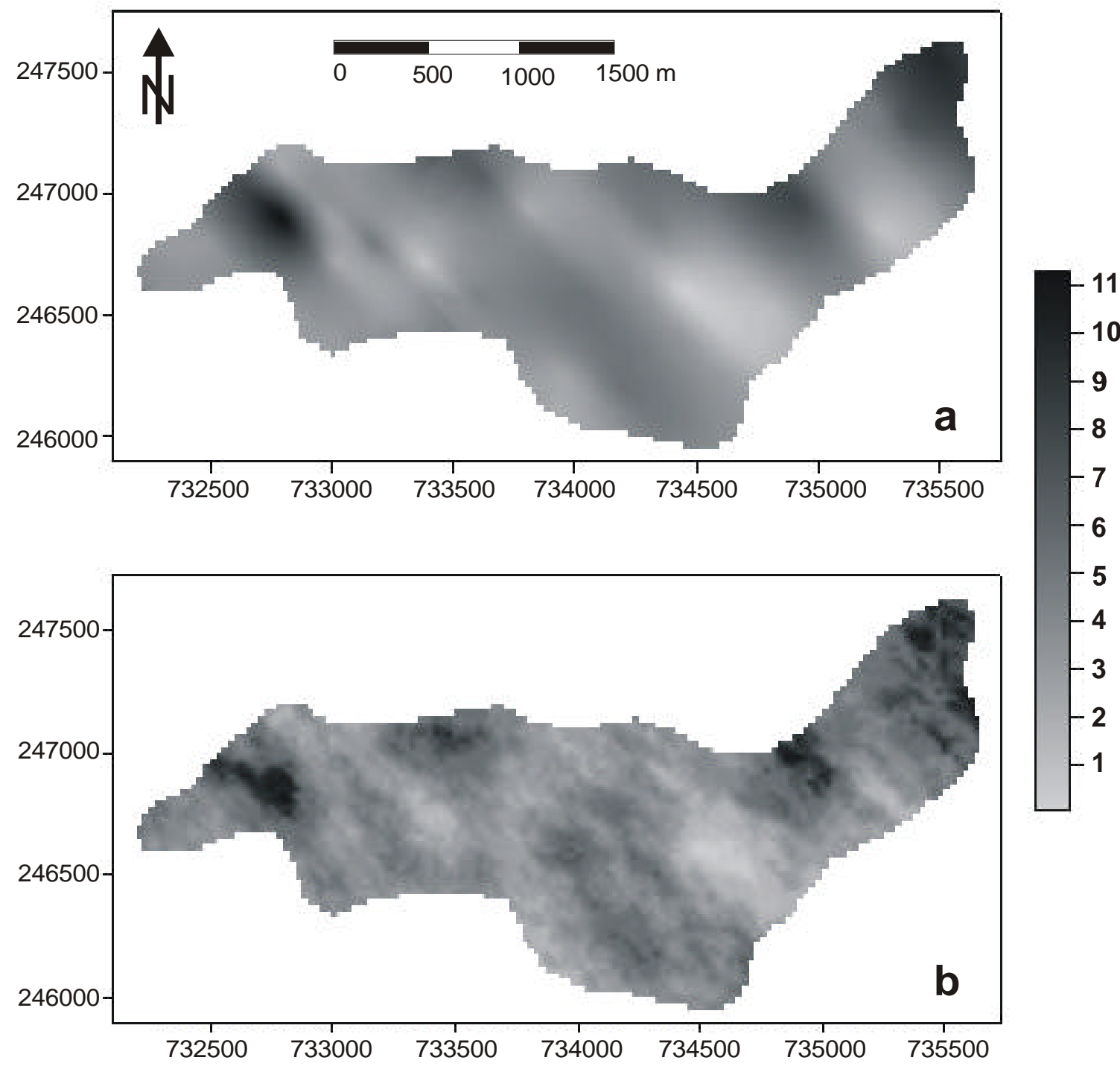

Figura 10. Mapa del comportamiento de la porosidad efectiva en el horizonte Tobas Gruesas del yacimiento Pina. a) Estimaciones, b) Simulaciones. 
fracturación en la zona. Este estudio fue realizado con todas las variables que intervienen en la estimación de reservas de hidrocarburos, lo que permitió obtener los valores de reservas probadas para cada horizonte del yacimiento.

Es importante destacar que el mapa de estimaciones (Figura 10a) presenta un comportamiento suave, es decir, no se observan las fluctuaciones que en la generalidad de los casos están presentes en la práctica, este hecho se puede apreciar en la mapa de simulaciones (Figura 10b), donde los valores simulados fluctúan alrededor de los valores estimados en los correspondientes puntos del mapa de estimaciones. A las zonas de estimaciones altas, le corresponden también valores altos pero con mayor dispersión y del mismo modo en las zonas de valores bajos. Estos procedimientos indiscutiblemente permiten una mejor caracterización de las variables que se estudian en las Ciencias de la Tierra.

\section{Conclusiones}

En el proceso de simulación se obtiene una representación con iguales características de variabilidad y correlación espacial que la realidad estudiada. Este proceso se concentra fundamentalmente en la generación de valores a partir de una función de covarianza fija, obtenida del análisis estructural de la información experimental disponible, valores que posteriormente son condicionados a dicha información. La simulación, a diferencia de la estimación que proporciona una representación suave o lisa de la realidad, es considerada como una de las posibles realizaciones de una función aleatoria, de la cual la realidad se considera también como una realización particular.

El éxito de la simulación está dado en que permite mostrar una representación de la realidad, nunca conocida totalmente en la mayor cantidad de problemas prácticos de las geociencias. La simulación no proporciona el mejor valor a obtener en una localización, pero sí muestra lo que podría ser el fenómeno real de forma general, pues reproduce los dos primeros momentos experimentales (media y covarianza $\mathrm{C}(\mathrm{h})$ o Semivariograma $\gamma(\mathrm{h})$ ) de los datos reales disponibles, así como su histograma.

No se debe distinguir entre simulación o estimación como dos procedimientos que se contradicen, aunque sus objetivos son diferentes, pero dependiendo de los intereses particulares en una aplicación o investigación se deben usar como dos alternativas que complementan un estudio: la simulación para obtener una representación del fenómeno de forma general y la estimación para obtener el mejor valor estimado en cualquier localización puntual o de bloque. Se presenta el comportamiento de la porosidad efectiva del horizonte Tobas Gruesas del yacimiento Pina de la provincia Ciego de Ávila, en el cual se pueden observar los resultados de la aplicación de estas dos herramientas, que en lugar de representar una contradicción, son dos formas diferentes para la caracterización de parámetros en las Ciencias de la Tierra.

Los resultados obtenidos en la simulación de la porosidad efectiva permitió reproducir el comportamiento de esta variable en horizontes productivos, sin necesidad de tener que realizar otros análisis geofísicos o de núcleos. Un estudio similar fue desarrollado en tres dimensiones, en el cual, a través de los cortes por niveles y perfiles, es posible profundizar en el conocimiento de la distribución de la variable en el área, correlacionándola con las direcciones de máxima fracturación, para proponer zonas con mejores caracte-rísticas de los colectores $\mathrm{y}$, consecuentemente, con mayores probabilidades de existencia de acumulaciones de hidrocarburos.

\section{Referencias bibliográficas}

Allard, D., 1993, Simulation du modèle gaussien seuillé conditionné par des contraintes de connexité: Compte-Rendu des Jounées de Géostatistique, p. 21-34.

Álvarez, J., Reyes, O., Segura, R., 1996, Modelo geológico del yacimiento Pina: Ciudad de la Habana, Cuba, Centro de Investigaciones del Petróleo.

Armstrong, M., Carignan, J., 1997, Géostatistique linéaire, application au domaine minier: Paris, France, École de Mines de Paris, France, $112 \mathrm{p}$.

Chauvet, P., 1994, Aide-memoire de géostatistique miniere: Paris, École des mines de Paris, France, 210 p.

Chica-Olmo, M., 1987, Análisis geoestadístico en el estudio de la explotación de recursos minerales: Granada, España, Universidad de Granada, Tesis Doctoral, 387 p.

Christakos, G., Panagopoulos, C., 1992, Space transformation methods in the representation of geophysical random fields: IEEE Transformation on Geosciences and Remote Sensing, 30 (1), 55-70.

Deutsch, C. V., Journel, A. G., 1998, GSLIB, Geostatistical Software Library and User's Guide, Second edition: Oxford University Press, 369 p.

Dietrich, C.D., 1995, A simple and efficient space domain implementation of the turning bands methods: Water Resources Research, 31 (1), 147-156.

Galli, A., 1997, Stochastic models for reservoir characterization: a user-friendly review, in 5th American and Caribean Petroleum Engineering Conference and Exhibition: Rio de Janeiro, Brazil, $11 \mathrm{p}$.

Guibal, D., 1972, Simulation de schémas intrinséques: Fontainebleau, France, École des Mines de Paris, Centre de Géostatistique, N291, 38 p.

Journel, A.G., 1974, Simulation conditionnelle de gisements miniers Theorie et Pratique: Paris, École des Mines de Paris, Thèse Docteur-Ingénieur, $110 \mathrm{p}$.

Journel, A.G., Huijbregts, C.J., 1978, Mining Geoestatistics: London, Academic Press, 600p.

Lantuéjoul, Ch., 1994, Non conditional simulation of stationary isotropic multigaussian random functions, in Armstrong, M., Dowd, P.A. (eds.), Geostatistical Simulation: Proceeding of the Geoestatistical Simulation Workshop, Fontainebleau, France, 27 28 may 1993, Dordrecht, Kluewer, p. 147-177.

Lantuéjoul, Ch., 1997, Interactive algorithms for conditional simulations, in Baah, E. Y., Ernest, Y., Schohald, N.A. (eds.), Geostatistics Wollongong'96: Dordrecht, Kluwer, 1, 27-40.

Lantuéjoul, Ch., 1998, Notas de clases del curso simulación, en el entrenamiento de postgrados, Ciclo de formación de especialistas en geoestadística, Curso 1997-98: Fontainebleau, France, Centro 
de Geoestadística de la Escuela Superior de Minas de Paris, 12 p.

Le Loc'h, G., Galli, A., 1997, Truncated plurigaussian methods: theoretical and practical point of view, in Baah, E.Y., Ernest, Y., Schohald, N.A. (eds.), Geostatistics Wollongong'96: Dordrecht, Kluwer, 1, 211-222.

Matheron, G., 1970, La téorie des variables régionalisées, et ses applications: Les Cahiers du Centre de Morphologie Mathématique de Fontainebleau, núm. 5, 112 p.

Myers, D. E., 1988, Multivariable geostatistical analysis for environ- mental monitoring, geomathematical and geostatistics analysis applied to space and time dependent data: Nancy, France, Science de la Terre, 27, 411-427.

Rivoirard, J., 1998, Notas de clases del curso simulación, en el entrenamiento de postgrados, Ciclo de Formación de Especialistas en Geoestadística, Cursos: 1995-96 y 1997-98: Fontainebleau, France, Centro de Geoestadística de la Escuela Superior de Minas de Paris, 12 p. 